\title{
An Adaptive Multiresolution-Based Multispectral Image Compression Method
}

\author{
Jonathan Delcourt, Alamin Mansouri, Tadeusz Sliwa, and Yvon Voisin \\ Laboratoire Le2i, BP 16 Route des Plaines de l'Yonne, 89010 Auxerre Cedex, France \\ jonathan.delcourt@u-bourgogne.fr
}

\begin{abstract}
This paper deals with the problem of multispectral image compression. In particular, we propose to substitute the built-in JPEG 2000 wavelet transform by an adequate multiresolution analysis that we devise within the Lifting-Scheme framework. We compare the proposed method to the classical wavelet transform within both multi-2D and full$3 \mathrm{D}$ compression strategies. The two strategies are combined with a PCA decorrelation stage to optimize their performance. For a consistent evaluation, we use a framework gathering four families of metrics including the largely used PSNR. Good results have been obtained showing the appropriateness of the proposed approach especially for images with large dimensions.
\end{abstract}

\section{Introduction}

Multispectral images are widely used in geoscience and remote sensing. Their use in other applications field like medical imagery, quality control in industry, meteorology, and exact color measurements are increasing. A multispectral image is generated by collecting dozens, so-called image-bands, where each one is a monochromatic image centered on a particular wavelength of the electromagnetic spectrum. As a result, uncompressed multispectral images are very large, with a single image potentially occupying hundreds of megabytes. Compression is thus necessary to facilitate both the storage and the transmission.

One of the most efficient compression method for monochrome images is the JPEG 20001 [1-5]. However, its extension to multispectral images needs to be adapted and yields to different methods. These methods depend on the manner of which one consider the multispectral cube:

- each image band of the multispectral image is considered separately (2D wavelets +2 D SPIHT),

- the whole cube is considered as input (3D wavelets $+3 \mathrm{D}$ SPIHT).

The contributions of this paper consists in substituting the built-in JPEG 2000 wavelet transform by an adaptive multiresolution analysis that we develop within the Lifting-Scheme framework. We will compare this approach with the standard version of JPEG 2000 within the previous presented strategies.

${ }^{1}$ http://www.jpeg.org

A. Elmoataz et al. (Eds.): ICISP 2010, LNCS 6134, pp. 54 62, 2010.

(C) Springer-Verlag Berlin Heidelberg 2010 
In the next section we will develop the theory of the proposed multiresolution analysis. Then we describe the data, experiments and results before discussing them in the third section. We concluded in the last section.

\section{Proposed Multiresolution Analysis}

We propose to substitute the wavelet transform by building an adequate multiresolution analysis adapted to the image compression aim. We build our multiresolution analysis within the lifting-Scheme framework which allows to produce easily second generation multiresolution analyses [6, 7]. Indeed, the advantage of such framework is that the inverse transform is explicitly defined and its value is exactly computed, whatever are the applied filters. We limit here the demonstration and illustration to the case of a scheme built on a couple of filters, one for prediction and one for update. The idea we aim to exploit consist in adapting the predict filter for each stage of the analysis in that way we minimize the energy hold by the detail signal and reduce more again the non-zero elements after the quantization stage. However, we risk in doing so to lose the error propagation control during the inverse transform. Indeed, ideal compression requires building perfect orthogonal transforms. But when we generate adaptive filters we cannot guarantee the orthogonality condition nor to be near. In order to reduce such a problem effect, we add a term expressing the constraint of near-orthogonality that we resolve using an optimization under constraints method.

We recall that we work within a scheme with one predict filler $f$ and one update filter $g$. The linear operator corresponding to the scale change and its inverse could be written as [8, 9]:

$$
\left(\begin{array}{l}
A \\
D
\end{array}\right)=\left(\begin{array}{cc}
a I & 0 \\
0 & b I
\end{array}\right)\left(\begin{array}{ll}
I & g \\
0 & I
\end{array}\right)\left(\begin{array}{cc}
I & 0 \\
-f & I
\end{array}\right)\left(\begin{array}{l}
O \\
E
\end{array}\right),\left(\begin{array}{l}
O \\
E
\end{array}\right)=\left(\begin{array}{ll}
I & 0 \\
f & I
\end{array}\right)\left(\begin{array}{cc}
I & -g \\
0 & I
\end{array}\right)\left(\begin{array}{cc}
\frac{1}{a} I & 0 \\
0 & \frac{1}{b} I
\end{array}\right)\left(\begin{array}{l}
A \\
D
\end{array}\right)
$$

Where $A$ is the approximation signal, $D$ the details signal, $O$ and $E$ the odd and even signals, $a$ and $b$ normalization coefficients (that we can consider positive), $I$ the identity matrix, and $f$ and $g$ are assimilated to their form of Toeplitz matrices.

The non-orthogonality condition of a linear operator $T$ could be written worthily as following:

$$
\varepsilon_{1}=T^{*} T-I, \varepsilon_{2}=T^{*}-T^{-1}, N\left(\varepsilon_{k}\right) \leq c
$$

Where $N$ is a matrix norm and * represents the conjugate of the transpose operation. In the case of a filter with real values, the ${ }^{*}$ is the convolution filtering. Let us also notice that the product of two filters is commutative due to the commutativity of the convolution product.

One important mathematical property we will exploit is the equivalence between all norms in finite dimension. This is our case since the number of samples is finite. Hence we will define and use a norm that ensures simplification 
of calculus in (2). First, equation (1) could easily be simplified as in $[8,9]$ by putting $b=1 / a$. Then after forward simplification of calculus in (2), it appears that we can make non-diagonal elements in $\varepsilon_{2}$ equal to zero when considering $g=f^{*} / a^{2}$. In doing so, we limit the solutions that minimize the nonorthogonality condition within the following family:

$$
b=\frac{1}{a}, g=\frac{1}{a^{2}} f^{*},\left(\begin{array}{l}
A \\
D
\end{array}\right)=\left(\begin{array}{cc}
a I-\frac{1}{a} f f^{*} & \frac{1}{a} f^{*} \\
-\frac{1}{a} f & \frac{1}{a} I
\end{array}\right)\left(\begin{array}{l}
O \\
E
\end{array}\right)
$$

We switch now to the definition $\varepsilon_{1}$ of non-orthogonality for calculus commodity. We inject $b$ and $g$ in $\varepsilon_{1}$ of equation (2) and we obtain a result proportional to:

$$
\left(\begin{array}{cc}
f f^{*}-\left(a^{2}-1\right) I & 0 \\
0 & f f^{*}-\left(a^{2}-1\right) I
\end{array}\right)\left(\begin{array}{cc}
f f^{*}-a^{2} I & f^{*} \\
f & I
\end{array}\right)
$$

By replacing $T$ by $T^{-1}$, we obtain a result proportional to:

$$
\left(\begin{array}{cc}
f f^{*}-\left(a^{2}-1\right) I & 0 \\
0 & f f^{*}-\left(a^{2}-1\right) I
\end{array}\right)\left(\begin{array}{lc}
I & f^{*} \\
f & f f^{*}-a^{2} I
\end{array}\right)
$$

The coefficient of proportionality is a common constant of value $1 / a^{2}$ meaning that it will not be involved in optimization.

Now we consider $N$ as the operator norm subordinate to the norm of Euclidean vector. In other words, for an operator $A$ :

$$
\|A\|_{2}=\sup _{v \neq 0} \frac{\|A v\|_{2}}{\|v\|_{2}}=\sqrt{\rho\left(A^{*} A\right)}=\sqrt{\rho\left(A A^{*}\right)}
$$

Where $\rho(M)$ represents the spectral ray of the matrix $M$, equivalent to the largest eigenvalue in absolute value.

From equations (4) and (5) one can remark that we can reason either on $T$ or $T^{-1}$. We can understand this intuitively as that the error propagates in the same way in direct and inverse sense.

By using the inequality of the product for the norm (6) in (4) and (5), we can evaluate independently the terms constituting the product.

The first term is completely determined by:

$$
f f^{*}-\left(a^{2}-1\right) I
$$

let us remark that (7D) is symmetric and we can reason on its eigenvalues. We can obtain the upper-bound for (6) applied to (7):

$$
\max \left(\left|\max \left(S p\left(f f^{*}\right)\right)-\left(a^{2}-1\right)\right|,\left|\min \left(S p\left(f f^{*}\right)\right)-\left(a^{2}-1\right)\right|\right)
$$

$S p$ corresponding to the spectrum. 
We consider now the second term of the product. After simplification using symbolic notations (Matlab symbolic toolbox), we can obtain, when we let $z_{\max }=\max \left(\left|\max \left(S p\left(f f^{*}\right)\right)-\left(a^{2}-1\right)\right|,\left|\min \left(S p\left(f f^{*}\right)\right)-\left(a^{2}-1\right)\right|\right)$, an upperbound for the second term as:

$$
\frac{z_{\max }+\sqrt{z_{\max }^{2}+4 a^{2}}}{2}
$$

Leading to the following upper-bounding for the product of the two terms:

$$
z_{\max } \frac{z_{\max }+\sqrt{z_{\max }^{2}+4 a^{2}}}{2}
$$

By identifying this upper-bound with the constraint $c$ in equation (2), we obtain:

$$
z_{\max }=\frac{c}{\sqrt{a^{2}+c}}=\frac{1}{a} \frac{c}{\sqrt{1+\frac{c}{a^{2}}}}<\frac{c}{a}
$$

the second term being equivalent to $\frac{c}{a}$ for its low values when $c$ tends to $0^{+}$.

For a constant value of $a$ and if we rename $c$ as $\frac{c}{a}$ in equation (11) we obtain:

$$
z_{\max }<c
$$

we find that all calculations are eventually determined by the spectrum of $\left(f f^{*}-\right.$ $\left.\left(a^{2}-1\right) I\right)$.

Beside, the norm mentioned in equation (6) is reduced to the spectral ray when the matrix is symmetric in one hand and the equivalence of norms in finite dimension allows us to use another norm $N$ to be upper-bounded in (2) in the other hand. Therefore, we can use as a norm $N$ the sum of the absolute values of the matrix coefficients, applied here to $\left(f f^{*}-\left(a^{2}-1\right) I\right)$.

We can now express the minimization under constraints for a filter having a support of length $n$ centered in the $\left(\frac{n}{2}\right)^{t h}$ coefficient as:

$$
\begin{aligned}
& \sum_{i=-\infty}^{\infty}\left(E_{i}-\sum_{k=-\frac{n}{2}+1}^{\frac{n}{2}} f_{k}\left(\tau_{-k} O\right)_{i}\right)^{2} \\
& -\lambda\left(\sum_{i=-\frac{n}{2}+1}^{\frac{n}{2}}\left|f_{i}^{2}-\left(a^{2}-1\right)\right|+2 \sum_{k=1}^{\frac{n}{2}}\left|\sum_{i=-\frac{n}{2}+1}^{\frac{n}{2}-k} f_{i} f_{i+k}\right|-c\right)
\end{aligned}
$$

Where $\tau$ is the translation operator, $i$ and $k$ the indices of both signals and filter coefficients $f$, and $\lambda$ a Lagrange coefficient.

The first term represents the prediction error (energy of the difference between Even $(E)$ and Odd $(O)$ signals after filtering) and the second term represents the constraint expressing the upper-bounding of the norm.

In practice for the orthogonality constraint, we have determined empirically the parameters values: $c=1.25 *\left(1-\frac{1}{n}\right), a=\sqrt{1+\frac{5}{n}}$. 
The size of the dictionary containing the filters increases proportionally to the number of scales and the length of the filters' supports. Which is why we limit experimentations only to short support filters $(n=2$ and $n=4)$. Furthermore, before storing each adaptive filter coefficients, we subtract from it an average fixed-values filter with the same length in such way to reduce its coefficients amplitudes. Afterwards, we use the maximal amplitude (negative or positive) that we encode in 2 bytes.

\section{$3 \quad$ Experiments and Results}

\subsection{Data and Experiments}

We conduct the experiments on 32 bands of the AVIRIS 2 Cuprite multispectral images with spatial dimensions of $64 * 64,128 * 128$ and $256 * 256$ pixels, coded in 16 bits integer. We aim to compare the performance of the proposed adaptive approach with the classical compression regarding different compression bitrates when using different spatial dimensions of images.

In order to optimize the multispectral image compression, a PCA (KLT) decorrelation step is used following spectral dimension. As a result, we obtain a new multi-band image in the transform domain in which the spectral correlation is reduced. We then applied the compression methods to the transformed image.

Two compression strategies are used in this article, Multi-2D and Full 3D. For 2D approach, each eigenimage from PCA is compressed separately. Afterwards, a 2D Set Partitioning In Hierarchical Trees 3 (SPIHT) coding [10] is applied on each band of the wavelets transform results to achieve compression. Because of PCA, the resulting image has decreasing energy bands. In order to take this fact into account, it is preferable to weight each band. As weights, we use the energy $E=\sqrt{\sum_{x, y} I_{\lambda}(x, y)^{2}} /(X Y)$. where $I_{\lambda}$ is the image band at the $\lambda$ wavelength, $X$ and $Y$ are its dimensions, and $x$ and $y$ are the position of a pixel in the band. Depending on energy band, we allocate proportional number of bits for the output of the SPIHT algorithm.

The 3D approach consist in considering the whole multispectral image cube as an input for a full 3D decomposition. To achieve compression a 3D SPIHT [11] is then applied.

\subsection{Compression Evaluation Framework}

When lossy compression methods are used, quality measurements are necessary to evaluate performance. In the case of ordinary 2D images, a metric has often to reflect the visual perception of a human observer. This is not the case for multispectral images, which are used for classification or spectral color reproduction. Which is why instead of evaluating compression performances according to one

${ }^{2}$ http://aviris.jpl.nasa.gov

${ }^{3}$ http://www.cipr.rpi.edu/research/SPIHT 
metric or one type of metric, we use four metrics (PSNR, spectral fidelity $\left(F_{\lambda}\right)$, universal image quality index (UIQI) and K-means classification driven metric) belonging to four families to evaluate performance as described in [12]. The aim of the proposed compression evaluation framework is to measure the performance of the compression strategies rather than quantifying distortions and noises produced during the compression process. Christophe et al. have studied and quantified such distortions in [13].

\subsection{Results and Discussion}

We compare the adaptive multiresolution proposed approach to the classical JPEG 2000 wavelets within two compression strategies (Multi-2D and Full 3D). We thus obtain four compression methods: Multi-2D (M2D), multiresolution Multi-2D (MR-M2D), Full 3D (F3D) and multiresolution Full 3D (MR-F3D) methods. We discuss separately methods belonging to the same strategy regarding different metrics. Figure 1 depicts the results in terms of PSNR and UIQI while the figure 2 gives compact representation containing all metrics for the bitrate of 1 bpp.

For the Multi-2D methods:

- In terms of PSNR and spectral fidelity, M2D method gives better results than MR-M2D method except for $64 * 64$ where this trend is inverted. The point of inversion is $\approx 0.7 \mathrm{bpp}$ for PSNR, and $\approx 0.5 \mathrm{bpp}$ for spectral fidelity.

- In terms of UIQI, M2D method gives worse results than MR-M2D. This metric is more based on structural distortion than error sensitivity.

- In terms of K-means, M2D and MR-M2D methods give quite similar results. Small differences can be noted for $128 * 128$ image size where M2D method have slightly better results than MR-M2D method for bitrate values upper than 0.25 bpp. This trend is inverted for $256 * 256$ image size.

For the Full 3D methods:

- For all images' sizes and for the majority of metrics MR-F3D gives quite better results than F3D. This difference of results are particularly high for small bitrates and increase with image's spatial dimensions.

- F3D method outperforms the MR-F3D only in terms of K-means for $256 * 256$ image size for bitrate values upper or equal at $0.5 \mathrm{bpp}$.

Within the Full 3D strategy, the proposed approach outperforms the classical one in almost all cases. We can highlight the fact that the goodness of results increases for small bitrates with the spatial dimensions of images: MR-F3D method has at least $\approx 0.5 \mathrm{~dB}$ higher than F3D method and at most $\approx 8 \mathrm{~dB}$. For high bitrates $(\approx 1 \mathrm{bpp})$ results become very close between the two methods.

Within the Multi-2D strategy, the M2D method performs slightly better than the MR-M2D. We can explain this by the fact that the multiresolution analysis creates a dictionary for each band of the image, leaving less bits for the SPIHT streams. The unique case in which MR-M2D outperforms M2D is in terms of UIQI, permitting to say that proposed method creates less structural distortions. 


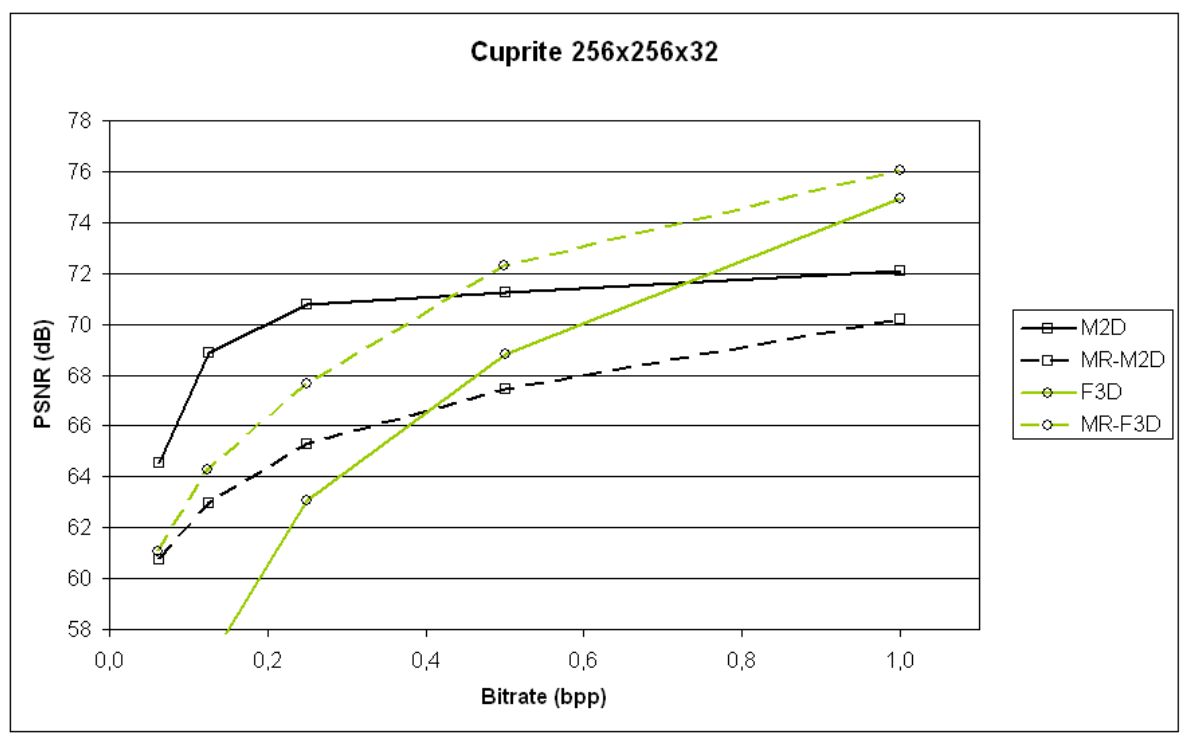

(a)

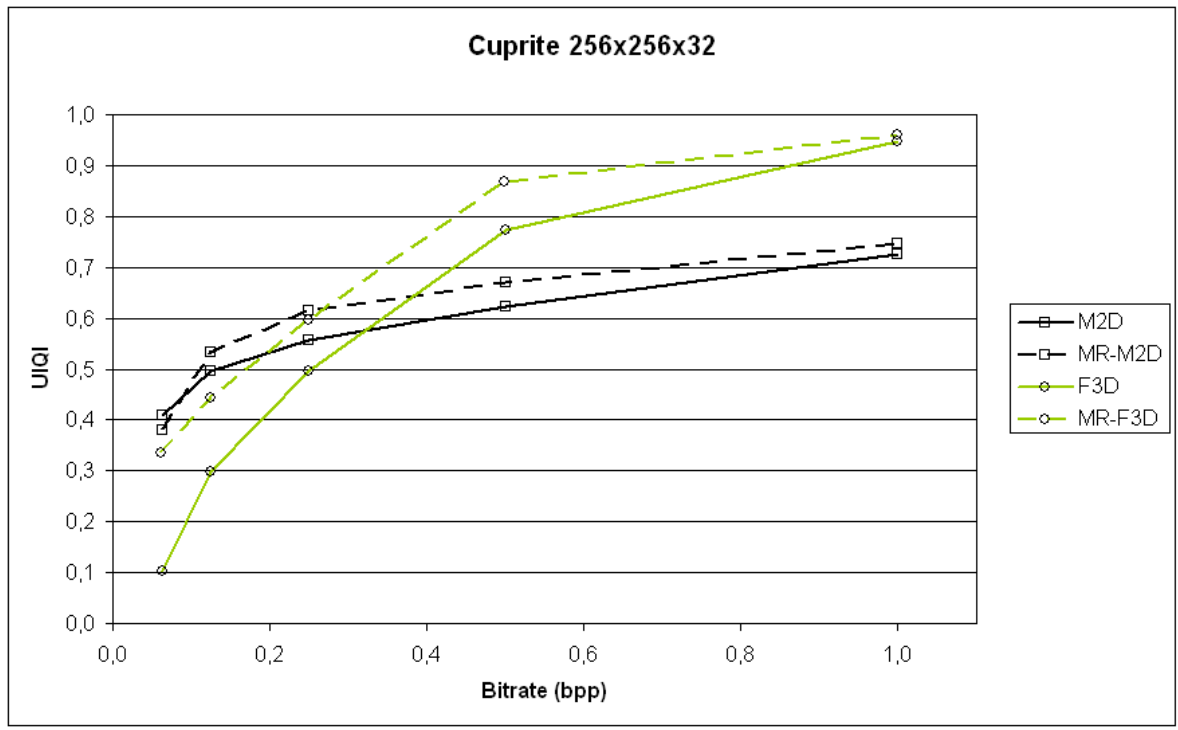

(b)

Fig. 1. Results of compression for Cuprite image of size $256 * 256$ in terms of (a) PSNR and (b) UIQI 


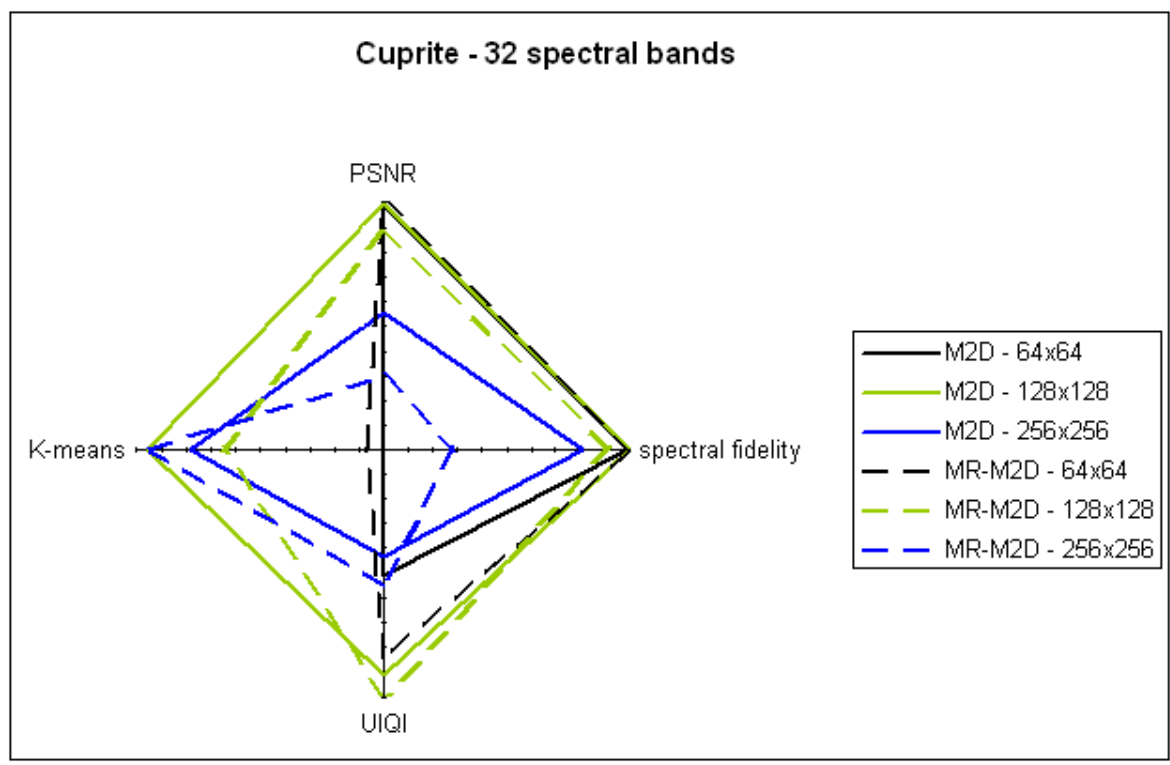

(a)

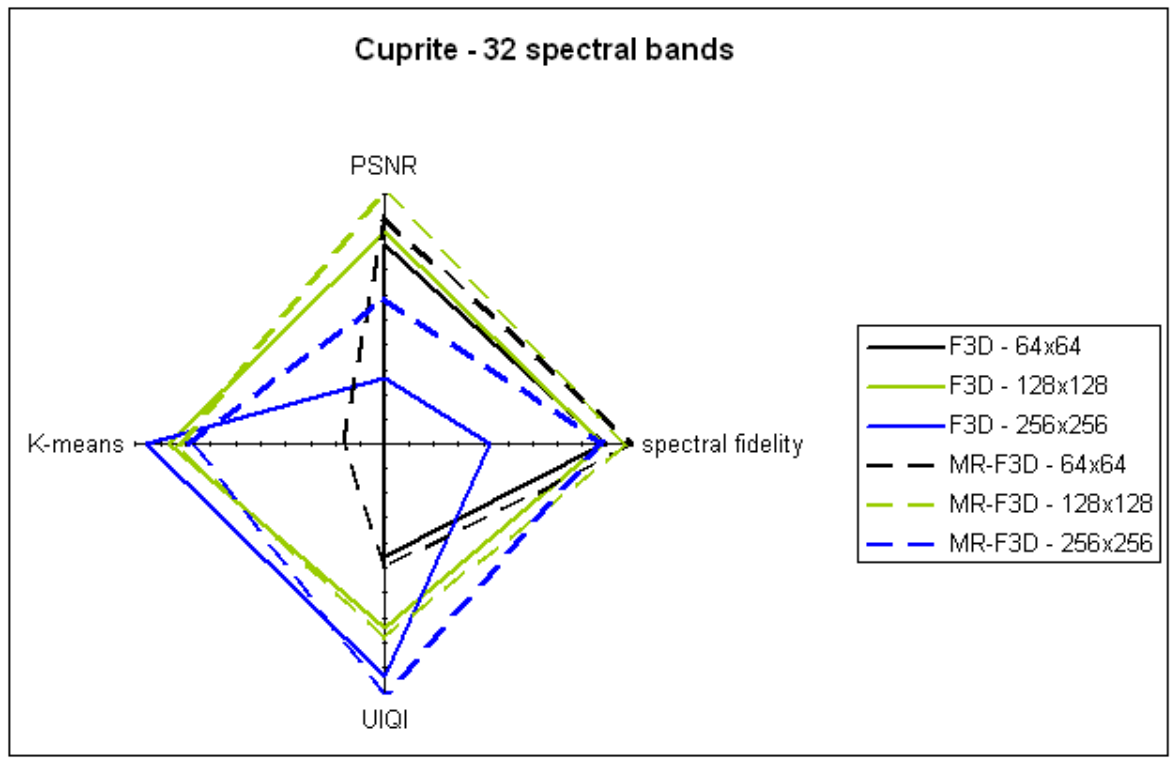

(b)

Fig. 2. Results of (a) M2D and (b) F3D strategies on Cuprite image for spatial dimension of $64 * 64,128 * 128$ and $256 * 256$ pixels in terms of PSNR, Spectral fidelity, UIQI and K-means 


\section{Conclusion}

In this work we introduced a new multiresolution analysis for multispectral image compression. This analysis was implemented within two compression methods: Multi-2D and Full 3D methods, and compared with the classical JPEG 2000.

The experiments showed the proposed approach is more appropriate for multispectral image compression especially within the Full 3D strategy for images with large spatial dimensions.

\section{References}

1. Boliek, M., Christopoulos, C., Majani, E.: JPEG 2000 Part I Final Committee Draft Version 1.0. ISO/IEC JTC, 1 (2000)

2. Boliek, M., Majani, E., Houchin, J.S., Kasner, J., Carlander, M.L.: JPEG 2000 part II final committee draft. ISO/IEC JTC1/SC29/WG1, FCD 15444, 2 (2000)

3. Christopoulos, C., Skodras, A., Ebrahimi, T.: The JPEG 2000 still image coding system: An overview. IEEE Transactions on Consumer Electronics 46(4), 1103-1127 (2000)

4. Taubman, D.: High performance scalable image compression with EBCOT. IEEE Transactions on Image Processing 9(7), 1158-1170 (2000)

5. Taubman, D.S., Marcellin, M.W., Rabbani, M.: JPEG2000: Image compression fundamentals, standards and practice. Journal of Electronic Imaging 11, 286 (2002)

6. Sweldens, W.: The lifting scheme: A construction of second generation wavelets. Technical Report, Department of Mathematics, University of South Carolina, 6 (1995)

7. Sweldens, W., Schroder, P.: Building your own wavelets at home. ACM SIGGRAPH course notes, 15-87 (1996)

8. Daubechies, I., Sweldens, W.: Factoring wavelet transforms into lifting steps. Journal of Fourier Analysis and Applications 4(3), 247-269 (1998)

9. Maslen, M., Abbott, P.: Automation of the lifting factorisation of wavelet transforms. Computer Physics Communications 127(2-3), 309-326 (2000)

10. Said, A., Pearlman, W.A.: A new, fast, and efficient image codec based on set partitioning in hierarchical trees. IEEE Transactions on circuits and systems for video technology 6(3), 243-250 (1996)

11. Dragotti, L., Poggi, G., Ragozini, A.R.P.: Compression of multispectral images by three-dimensional SPIHT algorithm. IEEE Transactions on Geoscience and Remote Sensing 38(1), 416-428 (2000)

12. Delcourt, J., Mansouri, A., Sliwa, T., Voisin, Y.: A comparative study and an evaluation framework of multi/hyperspectral image compression. In: 5th International Conference on Signal-Image Technology and Internet-Based Systems, SITIS 2009 (2009)

13. Christophe, E., Léger, D., Mailhes, C.: Quality criteria benchmark for hyperspectral imagery. IEEE Transactions on Geoscience and Remote Sensing 43(9), 2103 (2005) 